\title{
Long-Term X-ray Variability of Circinus X-1 as Observed by the $R X T E$ All Sky Monitor
}

\author{
Junfeng Wang and W. N. Brandt \\ Department of Astronomy and Astrophysics, Pennsylvania State \\ University, 525 Davey Lab, University Park, PA 16802, USA
}

\begin{abstract}
The luminous low mass X-ray binary Cir X-1 has been observed nearly continuously for about 5 years by the X-ray All Sky Monitor on board the $R X T E$ satellite. We carried out a timing analysis on Cir $\mathrm{X}-1$ with the $R X T E$ data. We define the period from the X-ray data, comparing the period change over time with the best current ephemeris. With folded light curves of entire data set, characteristics of the system like long-term lightcurve changes, behavior of flares and dips and superEddington accretion were obtained. We also checked and identified the secondary flaring reported in radio band with X-ray data.
\end{abstract}

\section{Introduction}

Cir X-1(4U 1516-56) is a bright, highly variable galactic X-ray source. It regularly exhibits flares in X-ray and radio bands, which is associated with the 16.55 day orbital period of the binary. The compact object in the binary is considered as a neutron star with the detection of type I X-ray bursts. The periodic Xray flares occuring near periastron can be explained as the results of enhanced accretion when neutron star is passing the periastron of a highly eccentric binary orbit. Fender (1997) reported a secondary radio outburst half an orbit after the primary flare for the first time, which is correlated with an increase in X-ray activity at this phase. The X-ray emission properties and the correlation with orbital phase show long-term changes as detected by Ginga ASM. Also near zero-phase the system exhibits intensity dips. Brandt et al. (1996) suggested that the dips can be explained by the change of partial covering fraction of absorbing matter and the true intensity may not be changed during the transition. Previous effort by Shirey et al. (1996) using $R X T E$ all-sky monitor (ASM) made three-month data of Cir X-1 available. Now since RXTE ASM has been nearly continuously observing Cir X-1 for five years, and its sensitivity apparently surpass Ginga ASM, we have been able to expect a more systematic, complete and accurate study of Cir X-1. New radio observation and long-term infrared monitoring work on Cir X-1 also call for a X-ray long-term study for this puzzling system. Our work can also be helpful to observation planning if the observer is interested in a certain phase of Cir X-1. 


\section{Observations and results}

The RXTE ASM results are provided by the ASM/RXTE teams at MIT and at the $R X T E$ SOF and GOF at NASA's GSFC. The data are quoted as nominal 2-10 keV rates in ASM counts per second. Our data covered from MJD 50088 to MJD 52263. A long-term decline in baseline intensity can be seen in the RXTE ASM counts curve after year 2000 , as shown in figure 1 . The ASM count rate of the base-level goes down from $\approx 90$ count $\mathrm{s}^{-1}$ to $\approx 40$ count $\mathrm{s}^{-1}$. One important feature we noticed is that the maximum flux is also shrinking at the same time. The count rate drops from $\approx 180$ count $\mathrm{s}^{-1}$ above the baseline level to $\approx 60$ count $\mathrm{s}^{-1}$. Similarly the dip intensity is also decreasing at the same time from $\approx 100$ count $\mathrm{s}^{-1}$ below the baseline level to $\approx 40$ count $\mathrm{s}^{-1}$.

We determined the period for Cir X-1 of $16.5608 \mathrm{~d}$ with two methods, namely Lomb-Scargle periodogram and Phase Dispersion Minimization. This means Xray flares onset occur regularly as in radio band. The folded light curves of the entire data set reveal that the dips always start at phase $0.9-1$ and $0-0.1$, the primary flares always occur at phase $0.1-0.3$ and the starting phases of secondary flares smear out in the range of phase $0.3-0.8$. A connection between dip strength and flare strength might be plausibly expected on physical grouds of accretion. The strength is defined as the integral of event intensity over the event duration time. We established a unique way to get the beginning and ending time for each integration. First we find the baseline intensity by sliding a window along the data set and calculating the mean value in this chunk of data. With the rms value, we performed $\sigma$-clipping to the whole data set and calculated the baseline intensity again in the same way. Then we define the starting point of the flare is where the count rate is above $3 \sigma$ level and the ending point is where the count rate drops below $3 \sigma$ level. And we add the phase as another constraint. We tried our best to find the correlation between dip strength and flare strength, but no strong correlation is detected. This can be interpreted as dip strength is not a good indicator of amount of the accreted material. We detect 82 secondary flares out of the 132 periods we examined. The distribution of the phases when secondary flares happen is not only between $0.6-0.8$, as Fender suggested, but also have a dispersion within $0.3-0.8$. Slightly rising in hardness ratio can be seen right before secondary flare, and then the hardness ratio decreases as secondary flare occurs. Even with a fairly small distance of $6.7 \mathrm{kpc}$ to Cir X-1, the $2-10 \mathrm{keV}$ luminosity is $L_{2-10} \geq 1.38 \times 10^{38}$ $\mathrm{erg} \mathrm{s}^{-1}$. During the flaring state, the count rate can be as high as 280 count s$^{-1}$, which is well above the count rate level corresponding to Eddinton luminosity of Cir X-1. The system must be experiencing super-Eddington accretion at phase $0.1-0.3$ when Cir X-1 is in high state. Considering only $2-10 \mathrm{keV}$ band, for at least $20 \%$ of the time Cir X-1 is in Super-Eddington accretion state.

\section{References}

Brandt, W. N. et al. 1996, MNRAS, 283, 1071

Fender, R. P. 1997, Proceedings of the Fourth Compton Symposium, AIP

Shirey, R. E., Bradt, H. V., Levine, A. M., \& Morgan, E. H., 1996, ApJ, 469, L21 